\title{
Estudio Comparativo de la Productividad y Calidad Obtenidas en la Soldadura de Tubos de Calidad T9 Empleados en el Sector Petroquímico, Mediante los Procesos TIG, HW-TIG y PAW.
}

\section{(Comparative Study of Productivity and Quality Obtained in Tube Welding Quality T9 Employees in the Petrochemical Industry, Through TIG, HW-TIG and PAW Processes)}

\author{
César García González ${ }^{1}$, Alejandro Conde Garrido ${ }^{1}$, Daniel Gestol ${ }^{1}$, Aurora López ${ }^{l}$ \\ ${ }^{1}$ Centro Tecnológico AIMEN, Planta de Tecnologías de la Unión, Porriño, Pontevedra, España, cggonzalez@aimen.es, aconde@ \\ aimen.es,dgesto@aimen.es,alopez@aimen.es
}

\begin{abstract}
Resumen
Tradicionalmente, la fabricación soldada de tuberías de acero Cr-Mo empleadas en serpentines de hornos para la industria petroquimica se ha venido realizando de forma masiva mediante procesos de baja productividad, principalmente TIG para la pasada de raiz y SMAW para las pasadas de relleno, ambos manuales. En este estudio se muestran los resultados de la investigación realizada por el Centro Tecnológico AIMEN, en relación al empleo de tecnologías de alto rendimiento, concretamente TIG, HW-TIG y PAW robotizadas, aplicadas a los mismos materiales y productos. De este modo se calculan y comparan cuatro índices de productividad: Tiempo de Soldadura, Tasa de Deposición, Material Depositado y Volumen de Gas Consumido. El objetivo del estudio es contribuir a la reducción de los tiempos de producción, de los costes de fabricación, y a la mejora de la calidad del producto.
\end{abstract}

Palabras-clave: $T I G, H W-T I G, P A W$, productividad,

\begin{abstract}
Traditionally, the manufacture of welded Cr-Mo steel pipes used in furnace coils for petrochemical industry has been carried out on a massive scale by low productivity processes, mainly TIG for root pass and SMAW for layers of filling run, both of them manuals. This study presents the results of a research, conducted by AIMEN Technology Centre, related to the use of high performance technologies, specifically robotic processes such as TIG, TIG-HW and PAW, applied to the same materials and products. Thus, four productivity ratios have been calculated and compared: welding time, deposition rate, deposited material and volume of consumed gas. The aim of the study is to contribute to the reduction of the production time, manufacturing costs and to the improvement of the product quality.
\end{abstract}

Key-words: $T I G, H W-T I G, P A W$, productivity

\section{Introducción}

Dentro de la industria petroquímica, los hornos de caldeo tienen la función de calentar el crudo o fracciones de éste como paso previo a su entrada en la torre de destilación donde se produce su fragmentación en hidrocarburos con diferentes temperaturas de ebullición. Tales hornos presentan una cámara interior en la que se monta un haz de tubos, en posición vertical, horizontal o en ambas, en forma de serpentín por el interior del cual circula el crudo. [1]

Los materiales seleccionados para los tubos que conforman los serpentines deben poseer una serie de características definidas por su entorno de trabajo, que se resumen fundamentalmente en: resistencia mecánica a elevadas temperaturas, resistencia al creep o termofluencia, resistencia a diferentes tipos de corrosión y oxidación y resistencia al desgaste. [2]

(Convidado em 15/05/2012; Texto final em 24/08/2012).
Teniendo en cuenta estos requisitos y considerando que los ataques por medios corrosivos y oxidantes son especialmente agresivos en entornos de temperaturas y presiones elevadas, la familia de aceros Cr-Mo encuentra en este sector un amplio campo de aplicación, especialmente los grados con alto contenido en cromo como las aleaciones $9 \% \mathrm{Cr}-1 \% \mathrm{Mo}$

Sin embargo, estas aleaciones presentan unos requerimientos de fabricación muy exigentes, debido a las precauciones que se deben tomar durante su soldadura para evitar la degradación de las propiedades del material, lo que obliga a la elección de procesos de soldeo de alta calidad y a la disposición de mano de obra altamente especializada.

La soldabilidad de los aceros $9 \% \mathrm{Cr}-1 \%$ Mo es dificultosa debido a la naturaleza alfágena de los elementos de aleación. Durante el enfriamiento, estos aceros son susceptibles de experimentar transformaciones martensíticas y, por tanto, son aceros fácilmente templables con propensión al agrietamiento por hidrógeno. Para evitarlo, se procede a un precalentamiento previo a la soldadura y a la ejecución de tratamientos térmicos postsoldadura (PWHT), que tratan de minimizar la formación o restituir esas fases frágiles. El empleo de gas de respaldo en las paradas de raíz está recomendado para evitar la oxidación en esta zona. [3] 
El proceso de soldeo más empleado hasta hoy para la soldadura de los tubos que conforman los serpentines es el TIG aplicado de manera manual. Entre los procesos de soldadura arco, el TIG/GTAW se caracteriza por su elevada calidad, baja productividad y su compleja aplicación. La soldadura TIG penaliza en gran medida la productividad y los costes de fabricación en espesores superiores a los $6 \mathrm{~mm}$, por lo que es frecuente su uso únicamente en las pasadas de raíz, empleando otros procesos, como el SMAW, en las pasadas de relleno.

Nuevos desarrollos automatizados de soldadura pueden aumentar de manera significativa la productividad, contribuyendo a la reducción de costes de fabricación sin afectar a la calidad final requerida a este tipo de uniones.

El objetivo del presente trabajo es evaluar, desde un punto de vista productivo, tres procesos de soldadura automatizada aplicables a la fabricación de los serpentines presentes en los hornos de caldeo.

Los procesos evaluados son el proceso TIG con aporte de hilo frío, el proceso HW (Hot wire)-TIG (que constituye un desarrollo del anterior en el que el hilo de aporte se calienta por medio de una resistencia eléctrica, lo que permite aumentar la Tasa de Deposición) y, finalmente, el proceso PAW con aporte de hilo frío, caracterizado por su elevado poder de penetración.

\section{Materiales y Métodos}

\subsection{Materiales}

\subsubsection{Material Base}

Dentro de la familia de aceros Cr-Mo, se seleccionó como material base el grado designado por la norma UNE EN 10216-2 como X11CrMo9-1 y por ASME como T9. [4,5]

Este grado de acero presenta un excelente comportamiento a fluencia y mayor resistencia a la corrosión que otros aceros Cr-Mo con menor contenido en cromo. El grado T9 es la aleación base de diferentes desarrollos de aceros $9 \% \mathrm{Cr}-1 \% \mathrm{Mo}$ modificados mediante la adicción de elementos de aleación formadores de carburos ( $\mathrm{Nb}, \mathrm{V}, \mathrm{N}, \mathrm{B}, \mathrm{W})$ y tratamientos térmicos específicos que incrementan tanto las propiedades mecánicas a temperatura elevada como su comportamiento a fluencia. Algunas de esas modificaciones son T91, T92, T991.

En la Tabla 1 se presenta la composición química de los tubos utilizados como material base, revelada mediante análisis químico por espectrometría de emisión por chispa de aleaciones de base hierro. Puede concluirse que esta composición entra dentro del rango marcado por la norma UNE EN 10216-2 para la aleación X11CrMo9-1. Las características mecánicas mínimas a temperatura ambiente para este acero, sometido a tratamiento térmico de normalizado y revenido, se definen en la misma norma y se presentan en la Tabla 2.

Este tipo de aleación es empleada en aplicaciones a elevada temperatura y condiciones de corrosión severas, siendo su temperatura de servicio máxima de $585^{\circ} \mathrm{C}$.
Tabla 1. Composición Química del Material Base

\begin{tabular}{|c|c|c|c|c|c|}
\hline $\mathrm{C}$ & $\begin{array}{c}0,130 \% \\
\pm 0,021\end{array}$ & $\mathrm{P}$ & $\begin{array}{c}0,020 \% \\
\pm 0,005\end{array}$ & $\mathrm{Cr}$ & $\begin{array}{l}8,430 \% \\
\pm 0,410 \\
\end{array}$ \\
\hline $\mathrm{Si}$ & $\begin{array}{c}0,327 \% \\
\pm 0,035\end{array}$ & S & $<0,010 \%$ & Mo & $\begin{array}{l}1,000 \% \\
\pm 0,066\end{array}$ \\
\hline Mn & $\begin{array}{c}0,471 \\
\pm 0,057\end{array}$ & $\mathrm{Ni}$ & $<0,370 \%$ & $\mathrm{Cu}$ & $\begin{array}{c}0,129 \% \\
\pm 0,041\end{array}$ \\
\hline
\end{tabular}

Tabla 2. Propiedades Mecánicas del Metal Base

\begin{tabular}{l|c}
\hline $\begin{array}{l}\text { Límite elástico convencional } \\
\mathrm{Rp} 0,2 \text { para un espesor } \mathrm{t} \leq 16\end{array}$ & $390 \mathrm{MPa}$ \\
\hline $\begin{array}{l}\text { Resistencia a tracción } \\
\mathrm{Rm}\end{array}$ & 580 a $740 \mathrm{MPa}$ \\
\hline $\begin{array}{l}\text { Alargamiento longitudinal } \\
\text { A min. }\end{array}$ & $18 \%$ \\
\hline
\end{tabular}

En cuanto a la soldabilidad de estas aleaciones, hay que tener en cuenta que para el cálculo de la temperatura de precalentamiento no es aplicable el método del carbono equivalente del Instituto Internacional de Soldadura (IIW), aplicable a los aceros al carbono. De este modo, es necesario acudir a códigos o normas que establezcan recomendaciones sobre la soldabilidad de estas aleaciones. En la Tabla 3 se presentan las temperaturas de precalentamiento recomendadas por UNE EN 1011-2, ASME VIII div. 2 y ASME B31.3. [6, 7, 8]

Tabla 3. Temperatura de Precalentamiento

\begin{tabular}{cc}
\hline UNE EN 1011-2 & $200^{\circ} \mathrm{C}$ \\
\hline ASME VIII div. 2 & $205^{\circ} \mathrm{C}$ \\
\hline ASME B31.3 & $177^{\circ} \mathrm{C}$ \\
\hline
\end{tabular}

El formato de las probetas soldadas consistió en tubos de $152.4 \mathrm{~mm}(6 ")$ de diámetro interior, $7,5 \mathrm{~mm}$ de espesor de pared y longitud entorno a los $200 \mathrm{~mm}$.

\subsubsection{Material de Aporte}

El material de aporte utilizado es el recomendado para la soldadura de aceros $9 \% \mathrm{Cr}-1 \% \mathrm{Mo}$. Se empleó un hilo de $1,2 \mathrm{~mm}$ de diámetro, clasificación AWS A5.28 ER 80 B8, EN ISO 21952A: G CrMo9. La composición química y las propiedades mecánicas certificadas por el fabricante del hilo se presentan en la Tabla 4 y Tabla 5 respectivamente.

Tabla 4. Composición Química del Material de Aporte

\begin{tabular}{cc|cc|cc|cc}
\hline $\mathrm{C}$ & $0,080 \%$ & $\mathrm{P}$ & $0,01 \%$ & $\mathrm{Mo}$ & $0,99 \%$ & $\mathrm{Nb}$ & $0,003 \%$ \\
\hline $\mathrm{Si}$ & $0,042 \%$ & $\mathrm{Cu}$ & $0,14 \%$ & $\mathrm{Al}$ & $0,013 \%$ & $\mathrm{Sb}$ & $0,001 \%$ \\
\hline $\mathrm{Mn}$ & $0,50 \%$ & $\mathrm{Ni}$ & $0,41 \%$ & $\mathrm{~V}$ & $0,020 \%$ & $\mathrm{Sn}$ & $0,004 \%$ \\
\hline $\mathrm{S}$ & $0,004 \%$ & $\mathrm{Cr}$ & $8,81 \%$ & $\mathrm{Zr}+\mathrm{Ti}$ & $0,001 \%$ & $\mathrm{As}$ & $0,006 \%$ \\
\hline
\end{tabular}


Tabla 5. Propiedades Mecánicas del Metal Depositado

\begin{tabular}{lc}
\hline Límite Elástico & $540 \mathrm{Mpa}$ \\
\hline Carga de Rotura & $670 \mathrm{MPa}$ \\
\hline Alargamiento & $23 \%$ \\
\hline
\end{tabular}

\subsection{Equipos Empleados}

Para las pruebas de soldadura realizadas en el presente estudio, se empleó como manipulador un robot $\mathrm{ABB}$ con mesa posicionadota IRBP-250A y controlador IRC 5 integrado con una fuente de potencia MagicWave 5000 de Fronius. Esta fuente de potencia incorpora un modulo alimentador de hilo, KD 7000D11 y un módulo Plasma para soldadura PAW. La fuente de potencia, TransPocket 1500TIG, empleada para calentar el hilo en el proceso HW-TIG es independiente de la primera.
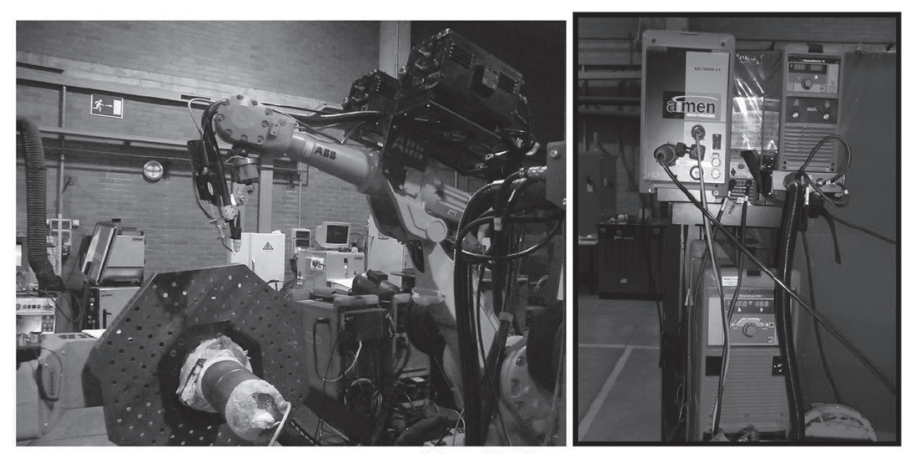

Figura 1. Robot de soldadura y equipo de soldeo

Para el precalentamiento de los tubos, se empleó un soplete portátil con acetileno como gas combustible y oxígeno como gas comburente. Para el control de la temperatura de precalentamiento se empleo un termómetro de contacto HIBOK 14, con un rango de temperaturas de -50 a $1300^{\circ} \mathrm{C}$ y precisión de \pm $\left(0,3 \%+1^{\circ} \mathrm{C}\right)$.

Una vez soldadas las probetas de estudio, se realizaron preparaciones metalográficas a partir de cortes transversales a los cordones de soldadura, y se procedió a su ataque por medio del reactivo Vilella para revelar las diferentes zonas de la soldadura: material base, zona afectada térmicamente (ZAT) y región fundida. Se empleó para la observación un microscopio metalográfico REICHERT MEF 4A n 20150

\subsection{Método Experimental}

El método experimental que se empleó, se puede dividir básicamente en los siguientes puntos:

a) Diseño de las uniones

b) Definición de los índices de productividad

c) Soldadura de probetas

\subsubsection{Diseño de las uniones}

El diseño de las uniones se varió en función del proceso de soldadura empleado para aprovechar las potenciales ventajas de cada uno de ellos. De este modo, para la aplicación del proceso TIG automatizado, se cerró el bisel respecto al habitualmente empleado en soldadura manual, en la que el soldador necesita mayor accesibilidad. En los tubos soldados por el proceso PAW el gap de la unión se redujo a cero para aprovechar la gran capacidad de penetración de este proceso. En la Figura 2 se presentan los diseños de junta empleados en las pruebas de soldadura, y se comparan con un diseño habitual en la soldadura manual.

\subsection{2. Índices de Productividad}

Como paso previo a la realización de las pruebas de soldadura, se fijan los índices técnicos que servirán de base para realizar una comparativa, desde el punto de vista de la productividad, entre los distintos procesos de soldadura. Estos factores son: Tiempo de Soldadura $\left(t_{\text {arco }}\right)$, Tasa de Deposición $(T D)$, Material Depositado $(M D)$ y Volumen de Gas Consumido $\left(V_{\text {gas }}\right)$.

El Tiempo de Soldadura $\left(t_{\text {arco }}\right)$ se define como el tiempo en el que el arco eléctrico permanece encendido. Viene determinado, directamente por la longitud de la unión $\left(L_{\text {sodada }}\right)$ y la velocidad de soldadura $\left(v_{\text {soldeo }}\right)$. Si se relaciona con el tiempo total en soldar una pieza se puede estimar el Factor de Operación del proceso.

$$
t_{\text {arco }}=\frac{L_{\text {soldada }}}{v_{\text {soldeo }}}
$$

Por Tasa de Deposición (TD) se entiende la cantidad de material depositado por unidad de tiempo de soldeo, generalmente es dada en $\mathrm{Kg} . \mathrm{h}^{-1}$. Para su cálculo se han de conocer: el diámetro del hilo de aporte $\left(\phi_{a}\right)$ en $\mathrm{m}^{2}$, la densidad del material de aporte $\left(\rho_{a}\right)$ en Kg. $\mathrm{m}^{-3}$, el rendimiento de deposición del aporte $\left(\eta_{a}\right)$ y la velocidad de alimentación del aporte $\left(v_{a}\right)$ en $\mathrm{m} \cdot \mathrm{min}^{-1}$.

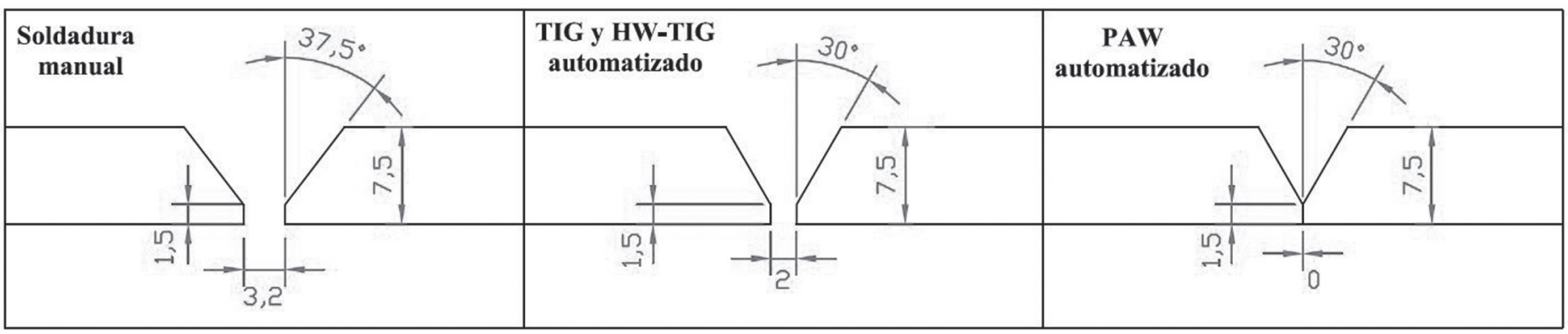

Figura 2. Preparaciones de unión empleadas en las pruebas 
$T D=60 \cdot \phi_{a} \cdot \rho_{a} \cdot \eta_{a} \cdot v_{a}$

El Material Depositado $(M D)$, es un factor clave para el cálculo de costes de soldadura y se define como la cantidad de material de aporte necesario para completar una unión determinada. Se calcula a partir de la tasa de deposición $(T D)$ y el tiempo de soldadura $\left(t_{\text {arco }}\right)$. Este índice suele darse en kilogramos.

$M D=\frac{T D \cdot t_{\text {arco }}}{60}$

Finalmente, el Volumen de Gas de Consumido $\left(V_{\text {gas }}\right)$ se define como la cantidad de argón empleada para completar la unión, en el presente trabajo este volumen se mide en litros. Para el cálculo de este índice se consideran: el caudal de gas de protección $\left(Q_{\text {gas }}\right)$, el caudal de gas de purga $\left(Q_{\text {purga }}\right)$, el tiempo de soldadura $\left(t_{\text {arco }}\right)$, el tiempo de soldeo de la pasada de raíz $\left(Q_{\text {raiz }}\right)$ durante el que se mantiene la purga y el tiempo de prepurga $\left(t_{\text {prepurga }}\right)$. En el caso del proceso PAW también se tiene en cuenta el caudal plasmágeno y su tiempo de aplicación.

$$
V_{\text {gas }}=Q_{\text {gas }} \cdot t_{\text {arco }}+Q_{\text {purga }} \cdot\left(t_{\text {raiz }}+t_{\text {prepurga }}\right)
$$

\subsubsection{Pruebas de Soldadura}

Una vez establecidos tanto el diseño de las uniones como los factores productivos a analizar, se procedió a la soldadura y registro de datos de las probetas mediante los procesos automatizados TIG, HW-TIG y PAW. Valores comunes a todas las pruebas de soldadura fueron la temperatura de precalentamiento, fijada finalmente en $200^{\circ} \mathrm{C}$, y el gas de protección: argón $99,90 \%$ de pureza. El caudal de purga $\left(Q_{\text {purga }}\right)$ se mantuvo en todos los casos en $201 . \mathrm{min}^{-1}$, con un tiempo de prepurga previo a la soldadura de 90 segundos, y se empleó corriente continua con polaridad directa.

En las soldaduras TIG y HW-TIG se empleó un electrodo de tungsteno aleado con un $2 \%$ de torio de $3,2 \mathrm{~mm}$ de diámetro y un afilado de $60^{\circ}$. La intensidad de calentamiento del hilo en el proceso HW-TIG se fijó en 110A. En la Tabla 6 y Tabla 7, se presentan los parámetros de soldadura empleados en ambos procesos.

Tabla 6. Parámetros soldadura TIG automatizado

\begin{tabular}{|c|c|c|c|c|}
\hline Pasada & $\begin{array}{l}\text { Intensidad } \\
\text { (A) }\end{array}$ & $\begin{array}{l}\text { Voltaje } \\
\text { (V) }\end{array}$ & $\begin{array}{c}\text { Vel. } \\
\text { soldeo } \\
\left(\mathrm{mm} \cdot \mathrm{s}^{-1}\right)\end{array}$ & $\begin{array}{c}\text { Vel. } \\
\text { hilo } \\
\left(\mathrm{m} . \mathrm{min}^{-1}\right)\end{array}$ \\
\hline Raíz & 145 & 10,0 & 1,1 & 1,0 \\
\hline Relleno & 175 & 10,1 & 2,0 & 1,7 \\
\hline Peinado & 200 & 12,3 & 1,6 & 1,7 \\
\hline
\end{tabular}

Tabla 7. Parámetros soldadura HW-TIG automatizado

\begin{tabular}{ccccc}
\hline Pasada & $\begin{array}{c}\text { Intensidad } \\
\text { (A) }\end{array}$ & $\begin{array}{c}\text { Voltaje } \\
(\mathrm{V})\end{array}$ & $\begin{array}{c}\text { Vel. } \\
\text { Soldeo } \\
\left(\mathrm{mm}^{-1} \mathrm{~s}^{-1}\right)\end{array}$ & $\begin{array}{c}\text { Vel. } \\
\text { Hilo } \\
\left(\mathrm{m}_{\mathrm{min}} \mathrm{min}^{-1}\right)\end{array}$ \\
\hline Raíz & 190 & 10,2 & 1,3 & 1,6 \\
\hline Peinado & 215 & 13,0 & 1,5 & 2,4 \\
\hline
\end{tabular}

Para la unión de los tubos mediante el proceso PAW se empleó argón como gas plasmágeno, con un caudal de 1,5 1. $\mathrm{min}^{-1}$ en la pasada de raíz y 0,6 1/min en el resto de la unión. Los parámetros utilizados se presentan en la Tabla 8.

Tabla 8. Parámetros soldadura PAW automatizado

\begin{tabular}{ccccc}
\hline Pasada & $\begin{array}{c}\text { Intensidad } \\
(\mathrm{A})\end{array}$ & $\begin{array}{c}\text { Voltaje } \\
(\mathrm{V})\end{array}$ & $\begin{array}{c}\text { Vel. } \\
\text { Soldeo } \\
\left(\mathrm{mm} \cdot \mathrm{s}^{-1}\right)\end{array}$ & $\begin{array}{c}\text { Vel. } \\
\text { Hilo } \\
\left(\mathrm{m} \mathrm{min}^{-1}\right)\end{array}$ \\
\hline Raíz & 165 & 18,3 & 0,85 & 0,5 \\
\hline Peinado & 155 & 20,1 & 1,5 & 1,5 \\
\hline
\end{tabular}

\section{Resultados y Discusión}

Tras una puesta a punto previa de parametrización, se obtuvieron uniones con buen aspecto visual. En las figuras 4-6 se muestran las macrografías y el aspecto de cada cordón de soldadura necesario para completar la unión, para los procesos TIG, HW-TIG y PAW respectivamente. Como puede observarse, en el caso de la soldadura TIG la unión se completó con tres cordones, mientras que en el caso de los procesos HW-TIG y PAW únicamente fueron necesarios dos.

\subsection{Comparativa factores de productividad}

Recopilados los tiempos y parámetros necesarios, y como paso previo a la estimación de los índices de productividad, se calcularon la densidad del material de aporte $\left(\rho_{a}\right)$ y el rendimiento de deposición del mismo $\left(\eta_{a}\right)$. La $\rho_{a}$ se calculó como la media de tres pesadas de hilos de un metro longitud, de manera que se obtuvo una densidad de $7555 \mathrm{Kg} \cdot \mathrm{m}^{-3}$.

Del mismo modo, el rendimiento de deposición $\left(\eta_{a}\right)$ se estima relacionando el peso real del cordón depositado, calculado de manera empírica a partir de pesajes de las probetas antes y después de la soldadura, y el peso teórico del cordón, calculado de manera analítica a partir de la densidad y el volumen del hilo introducido en la unión. El rendimiento de deposición medio obtenido para los tres procesos de soldeo fue del $95 \%$, lo que indica que las pérdidas de material de aporte por volatilización o proyecciones son reducidas.

Una vez conocidas todas las variables para la estimación de los índices de productividad, se procedió a su cálculo. En la tabla 9 se presentan estos índices para los tres procesos estudiados.

La reducción del tiempo de soldeo en el HW-TIG respecto al TIG con aporte de hilo frío es de un 30\%. El calentamiento del hilo permite aumentar la velocidad de alimentación y con ello la velocidad de soldadura, reduciendo tiempos. 

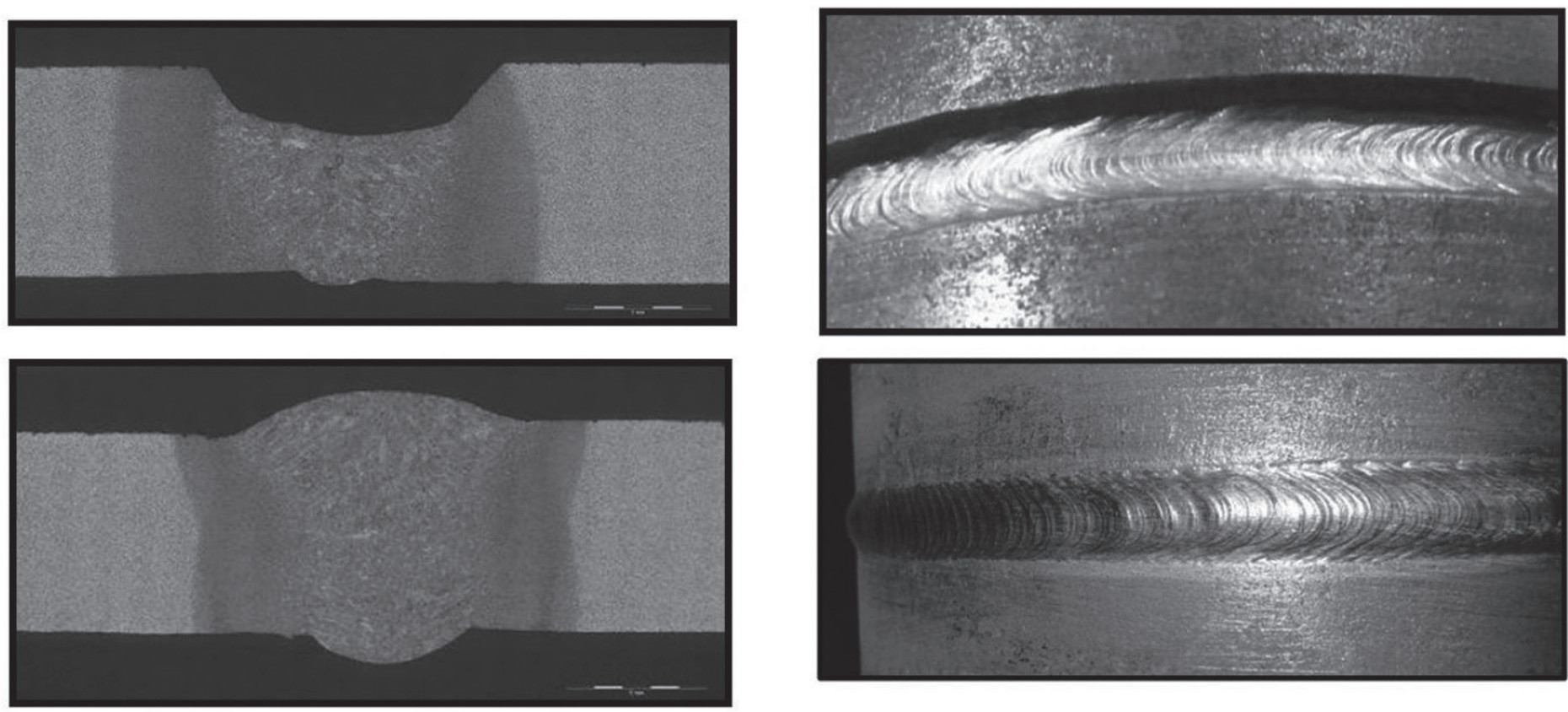

Figura 3. Macrografías y cordones depositados por el proceso TIG automatizado
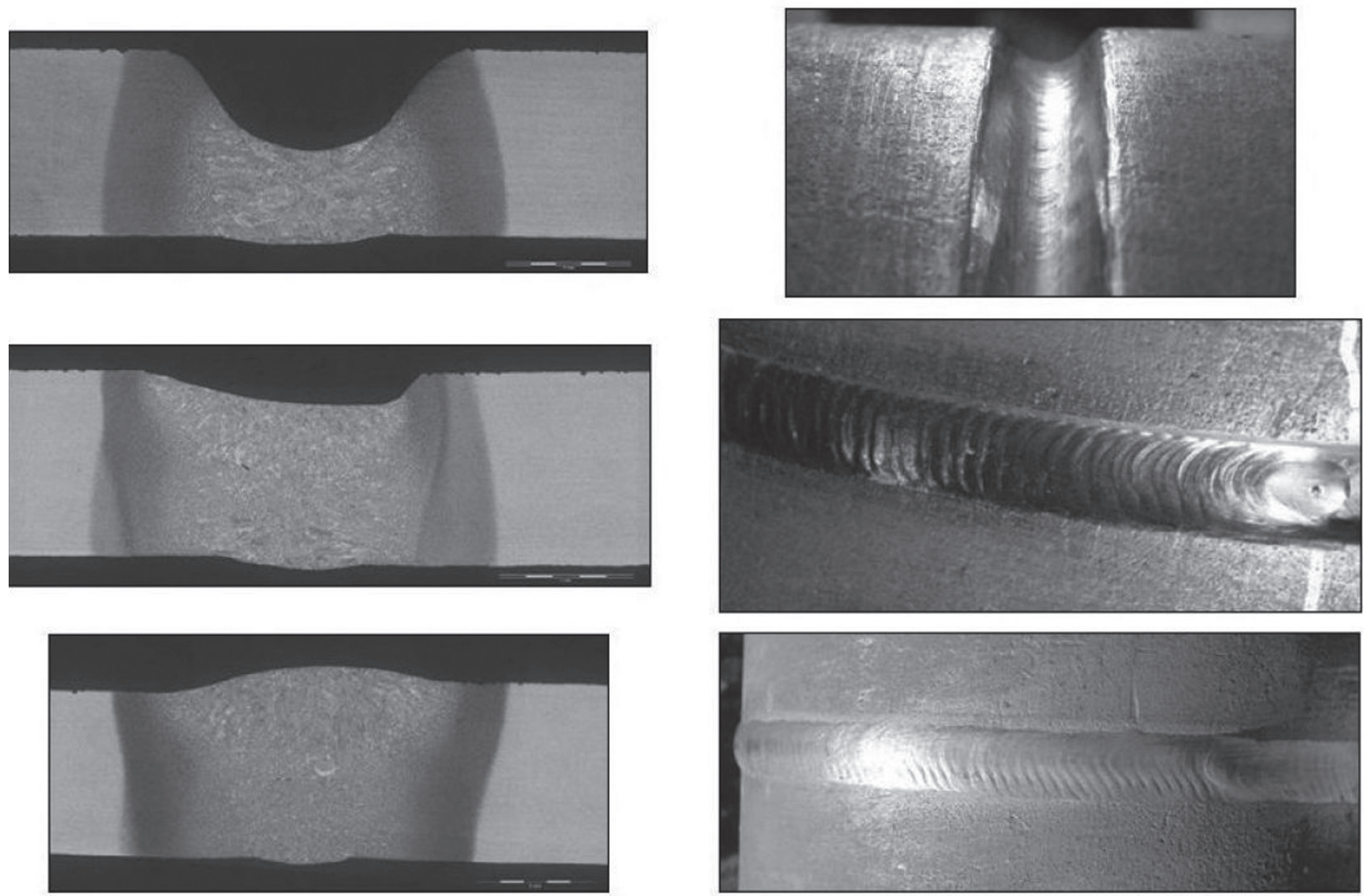

Figura 4. Macrografías y cordones depositados por el proceso HW-TIG 

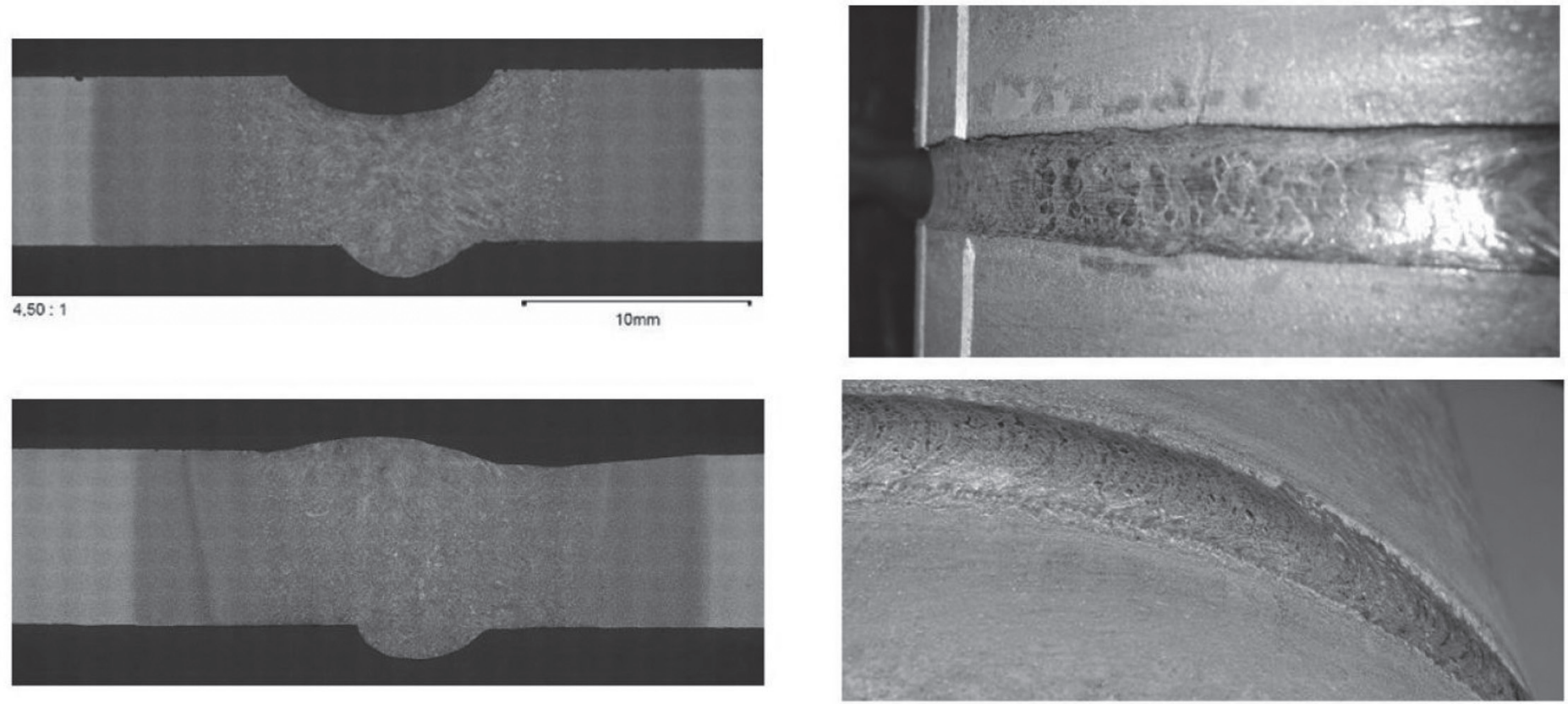

Figura 5. Macrografías y cordones depositados por el proceso PAW

En el proceso PAW, la reducción respecto al proceso TIG hilo frío es del $10 \%$ y se debe fundamentalmente a la reducción en el número de pasadas, ya que la preparación de la unión empleada en las soldaduras PAW, más cerrada que en los procesos TIG, obliga a reducir la velocidad de soldeo en la pasada de raíz.

Tabla 9. Índices de Productividad

\begin{tabular}{c|c|c|c}
\hline & TIG & HW-TIG & PAW \\
\hline $\begin{array}{c}\text { Tiempo de Soldadura } \\
(\mathrm{min})\end{array}$ & 16,83 & 11,8 & 15,15 \\
\hline $\begin{array}{c}\text { Tasa de Deposición } \\
\left(\mathrm{kg} \cdot \mathrm{h}^{-1}\right)\end{array}$ & 0,65 & 0,99 & 0,44 \\
\hline $\begin{array}{c}\text { Material Depositado } \\
(\mathrm{Kg})\end{array}$ & 0,18 & 0,19 & 0,11 \\
\hline $\begin{array}{c}\text { Gas consumido } \\
(\mathrm{l})\end{array}$ & 484 & 370 & 513 \\
\hline
\end{tabular}

Se puede apreciar cómo el efecto del calentamiento del hilo en el proceso HW-TIG, permite aumentar en 340 gramos el material depositado en una hora de soldadura, lo que supone un aumento en la tasa de deposición de un 52\%, respecto al TIG con aporte de hilo frío. De forma más visual, si se tiene en cuenta que una unión con la preparación utilizada para la soldadura TIG y HW-TIG, necesita aproximadamente 200 gramos de soldadura para completarse, y en el supuesto ideal de que el arco estuviera encendido una hora continua, se obtendrían:

- con el proceso TIG hilo frío: 3 uniones completas y un $25 \%$ de la cuarta.

- con el proceso HW-TIG: 4 uniones completas y el 95\% de la quinta.
En el caso del PAW, la menor cantidad de material aportado, junto con una moderada reducción del tiempo de soldeo, provoca que los cálculos realizados muestren una reducción en la tasa de deposición del 32\%, en comparación con el TIG hilo frío.

En cuanto al material depositado, el empleo de la misma preparación de la unión para los procesos TIG hilo frío y HW-TIG, lleva a que el material necesario para completar la unión sea el mismo, justificándose la pequeña diferencia en el peso por los diferentes sobreespesores de cara y de raíz obtenidos. En el caso de las uniones soldadas por el proceso PAW, la reducción en la sección de la preparación (Figura 2) se traduce en unas reducciones similares en el material de aporte empleado, un 39\%.

De forma global, respecto al TIG hilo frío se ahorra un 24\% en el gas consumido al utilizar el proceso HW-TIG debido a la reducción del número de pasadas, gracias a su mayor tasa de deposición. En el caso del proceso PAW, la baja velocidad de soldadura en la primera pasada, en la que se debe mantener un flujo constante de gas de purga, así como la necesidad de un determinado caudal para establecer y mantener el arco plasma, provoca un aumento del gas consumido del $6 \%$ respecto al proceso TIG con aporte frío.

\section{Conclusiones}

Después de analizar los distintos factores técnicos y de productividad resultantes de las pruebas realizadas, las principales conclusiones que se pueden extraer son:

a) La soldadura de las uniones contempladas en este estudio fueron completadas con dos pasadas para los procesos HW-TIG y PAW, lo que supone un cordón menos de los necesarios empleando el proceso TIG convencional.

b) El incremento de la tasa de deposición del proceso HW-TIG frente al TIG con aporte de hilo frío es de un $52.3 \%$, deri- 
vada de la posibilidad de trabajar con una mayor velocidad de alimentación de hilo, gracias a su mayor temperatura por precalentamiento.

c) En el proceso PAW se consigue un ahorro de material de aporte de un $39 \%$, gracias a su elevado poder de penetración, que posibilita trabajar con biseles más cerrados

d) El consumo de gas disminuye para el proceso HW-TIG en un $23 \%$, debido fundamentalmente a la disminución en el tiempo de soldeo necesario para cubrir la junta. En el proceso PAW, sin embargo, aumenta ligeramente, ya que el tiempo de soldadura se reduce de manera moderada respecto al TIG hilo frío, teniéndose que contabilizar, además, el caudal de gas necesario para establecer y mantener el arco plasma.

\section{Referencias Bibliográficas}

[1] PDVSA. Manual de Diseño de Proceso, Transferencia de Calor, Hornos. MDP-05-F-01 Principios Básicos. 1983. 61 p.

[2] PDVSA. Manual de Diseño de Proceso, Transferencia de Calor, Hornos. MDP-05-F-02 Consideraciones de Diseño. 1983. $64 \mathrm{p}$.

[3] OÑORO LÓPEZ, JAVIER. Aceros débilmente aleados resistentes a termofluencia. In: ASOCIACIÓN ESPAÑOLA DE SOLDADURA Y TECNOLOGÍAS DE UNIÓN. Formación de Ingenieros Internacionales de soldadura. 2004, Tema 2.14. 32 p. [4] AENOR. Tubos de acero sin soldadura para usos a presión. Condiciones técnicas de suministro. Parte 2: Tubos de acero no aleado y aleado con características a temperatura elevada. Madrid. 2008. 45 p.

[5] ASME. IX Welding and Brazing qualifications. 2007. $330 \mathrm{p}$. [6] AENOR. Soldeo. Recomendaciones para el soldeo de materiales metálicos. Parte 2: Soldeo por arco de los aceros ferríticos. UNE-EN 1011-2. Madrid. 2001. 63 p.

[7] ASME. VIII Division 2. Alternative rules: Rules for construction of pressure vessels. 2007. 998 p.

[8] ASME. Code for pressure piping, B31. ASME B31.3b-2001 Addenda to ASME B31.3-1999 edition process piping. 596 p. 